\title{
Automatic C to O-O Translation with C2Eiffel
}

\author{
Marco Trudel · Carlo A. Furia - Martin Nordio \\ Chair of Software Engineering, ETH Zurich, Switzerland \\ Email: firstname.lastname@inf.ethz.ch
}

\begin{abstract}
C2Eiffel is a fully automatic source-to-source translator of $\mathrm{C}$ applications into the Eiffel object-oriented programming language. C2Eiffel supports the complete $C$ language, including function pointers, unrestricted pointer arithmetic and jumps, arbitrary native libraries, and inlined assembly code. It produces readable Eiffel code that behaves as the source $\mathrm{C}$ application; it takes advantage of some of Eiffel's object-oriented features to produce translations that are easy to maintain and debug, and often even safer than their sources thanks to stricter correctness checks introduced automatically. Experiments show that C2Eiffel handles $\mathrm{C}$ applications of significant size (such as vim and $l i b g s l$ ); it is a fully automatic tool suitable to reuse $\mathbf{C}$ code within a high-level object-oriented programming language.
\end{abstract}

\section{Reusing C Code with a Click}

This paper presents C2Eiffel (C2Eif for short), a completely automatic tool that translates $\mathrm{C}$ source code into the Eiffel object-oriented programming language. With C2Eif, you can reuse $\mathrm{C}$ applications in a modern environment, where they can be seamlessly integrated with other native Eiffel code that fully takes advantage of the object-oriented paradigm and of other high-level language features such as contracts and static type safety. Since C2Eif translates source-to-source, the translated $\mathrm{C}$ code is not merely made available within a host environment-as is the case with integration solutions based on foreign language interfaces-but becomes a native Eiffel implementation, which developers can refactor and extend as the overall application evolves and undergoes maintenance. The translation provided by $\mathrm{C} 2$ Eif may even single-handedly improve the safety and debuggability of the $\mathrm{C}$ implementation, because it automatically introduces safety checks for issues such as out-of-bound array access and nullpointer dereferencing. The checks make understanding and debugging translated applications considerably easier, because faults manifest themselves closer to their source location, and because certain buffer overflow errors are harder to replicate and exploit within the tight Eiffel runtime.

Even if you are not developing applications in Eiffel, the same ideas used in C2Eif underlie the development of fully automatic translators of $\mathrm{C}$ into other full-fledged modern object-oriented programming languages such as Java and C\#. In fact, the chasm existing between the $C$ and Eiffel languages ensures that the translation of one into the other is not a simple transliteration (as it would have been possible, for example, with $\mathrm{C}++$ ) but has to provide output that is germane to the object-oriented paradigm.

C2Eif is open-source and available for download at http://se.inf.ethz.ch/research/c2eif

This tool demo paper describes the overall architecture of
C2Eif (Section III), presents the main results of an extensive experimental evaluation (Section III), and compares C2Eif against other similar tools (Section IV). This short paper only describes the tool architecture and usage; a detailed presentation of the translation scheme including examples is available in a companion paper [1].

\section{OvERVIEW AND ARCHITECTURE}

C2Eif is a compiler with a graphical user interface and a command-line interface that translates $\mathrm{C}$ programs to Eiffel. The translation produces a complete Eiffel application that is functionally equivalent to the $\mathrm{C}$ source application. C2Eif is implemented in Eiffel, and it is available as a standalone tool.

Input and output. Figure 1 shows the overall workflow of using C2Eif. It inputs $\mathrm{C}$ projects (applications or libraries) preprocessed with the $\mathrm{C}$ Intermediate Language (CIL) framework [2]. CIL simplifies programs written in $\mathrm{C}$ into a subset of $\mathrm{C}$ amenable to program transformation. Using CIL input ensures complete support of the whole set of $\mathrm{C}$ statements, without polluting the translation with variants and special cases only to deal with syntactic sugar. C2Eif translates CIL programs to Eiffel projects consisting of collections of classes.

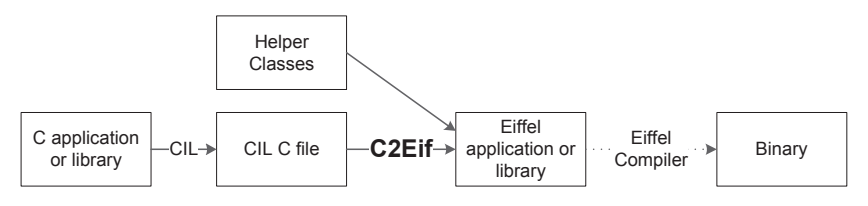

Fig. 1. Overview of how C2Eif works.

Incremental translation. C2Eif builds an initial AST by parsing the input $\mathrm{C}$ program, and transforms it into a target AST that can be pretty-printed as Eiffel code. Following a modular design that improves maintainability, C2Eif implements the translation from $\mathrm{C}$ to Eiffel as a series of successive incremental transformations on the AST. Every transformation targets exactly one language aspect (for example, loops or inlined assembly code) and produces an AST that combines C features with Eiffel extensions: the code progressively morphs from $\mathrm{C}$ to Eiffel. C2Eif can output the AST in text form after any of the intermediate transformations; the output is a mixture of $\mathrm{C}$ and Eiffel code that is readily understandable by programmers familiar with both languages. The current implementation uses about 40 transformations. 


\begin{tabular}{lrrrrr} 
& \multicolumn{2}{c}{ SizE (LOCS) } & \#EIFFEL & TRANSLA- & BINARY \\
& CIL & EIFFEL & CLASSES & TION (S) & SIZE (MB) \\
\hline hello world & 8 & 15 & 1 & 1 & 1.3 \\
micro httpd & 565 & 1,934 & 16 & 1 & 1.5 \\
xeyes & 1,463 & 10,661 & 78 & 1 & 1.8 \\
less & 16,955 & 22,545 & 75 & 5 & 2.6 \\
wget & 46,528 & 57,702 & 183 & 25 & 4.5 \\
links & 70,980 & 100,815 & 211 & 33 & 13.9 \\
vim & 276,635 & 395,094 & 663 & 144 & 24.2 \\
\hline libcurl & 37,836 & 65,070 & 289 & 18 & - \\
libgmp & 61,442 & 79,971 & 370 & 21 & - \\
libgs & 238,080 & 344,115 & 978 & 85 & - \\
\hline gcc (torture) & 147,545 & 256,246 & 2,569 & 79 & 1,576 \\
\hline ToTAL & 898,037 & $1,334,168$ & 5,433 & 413 & 1,626
\end{tabular}

TABLE I

AUTOMATED TRANSLATION OF OPEN-SOURCE PROGRAMS.

\section{EVALUATION}

Table II shows data about 10 open-source $\mathrm{C}$ programs and one testsuite (for $\mathrm{gcc}$ ) translated to Eiffel with C2Eif. The 10 programs include 7 applications and 3 libraries; all of them are widely-used in Linux and other *nixes. For each entry Table I reports: the size (in lines of code) of the CIL version of the $\mathrm{C}$ code and of the translated Eiffel code; the number of Eiffel classes created; the time (in seconds) spent by C2Eif to perform the source-to-source translation (not including compilation from Eiffel source to binary); the size of the binaries (in MBytes) generated by EiffelStudid ${ }^{1}$

We ran extensive trials on the translated programs to verify that they behave as in their original $\mathrm{C}$ version. In addition to informal usage, we ran standard testsuites on the 3 libraries totalling over 770 tests. All tests execute and pass on both the $\mathrm{C}$ and the translated Eiffel versions of the libraries, with the same logged output. The gcc torture testsuite includes 1002 tests that pass after being processed by CIL; C2Eif (which depends on CIL) passes 989 (nearly 99\%) and fails 13 of these tests (see [1] for a detailed discussion of the reasons for the 13 failures). Given the variety of the programs considered (and the challenging nature of the torture testsuite), the experiments are strong evidence that $\mathrm{C} 2 \mathrm{Eif}$ handles the complete $\mathrm{C}$ language used in practice, and produces correct translations.

Performance. We performed systematic performance tests for some of the applications; the results of the tests are described in detail in [1]. In summary, the performance overhead in switching from $\mathrm{C}$ to Eiffel significantly varies-from negligible to noticeable-with the program type. Even in the cases where the slowdown is noticeable, it does not preclude the usability of the translated application or library in normal conditions.

Safety. While running the Eiffel translation of libgmp we detected 3 bugs ( 1 in the library itself and 2 in the accompanying testsuite) thanks to the dynamic checks introduced automatically by C2Eif. More generally, C2Eif translations are often more robust and easier to debug than the original $\mathrm{C}$ versions thanks to the contracts introduced by $\mathrm{C} 2$ Eif and by the tighter Eiffel runtime.

\footnotetext{
${ }^{1}$ We do not give a binary size for libraries, because EiffelStudio cannot compile them without a client.
}

\begin{tabular}{|c|c|c|c|c|c|c|c|c|}
\hline & 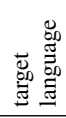 & 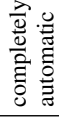 & $\begin{array}{l}\frac{0}{0} \\
\frac{\pi}{\pi} \\
\frac{\pi}{\pi}\end{array}$ & 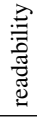 & 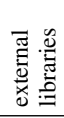 & 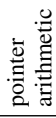 & 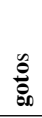 & 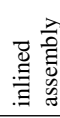 \\
\hline C2Eif & Eiffel & yes & yes & + & yes & yes & yes & yes \\
\hline Ephedra 3 & Java & no & no & + & no & no & no & no \\
\hline $\mathrm{C} 2 \mathrm{~J}++4$ & Java & no & no & + & no & no & no & no \\
\hline $\mathrm{C} 2 \mathrm{~J} 5$ & Java & no & yes & - & no & yes & no & no \\
\hline $\mathrm{C}++2$ Java 6 & Java & no & yes & + & no & no & no & no \\
\hline$C++2 C \# 6$ & $\mathrm{C \#}$ & no & yes & + & no & no & no & no \\
\hline
\end{tabular}

TABLE II

TOOLS TRANSLATING C TO O-O LANGUAGES.

\section{RELATED WORK}

A number of tools are available that translate $\mathrm{C}$ to objectoriented languages. Table II shows a summary feature comparison among the currently available tools. For each tool, Table II reports:

- The target language: Eiffel, Java, or C\#.

- Whether the tool is completely automatic, that is whether it generates translations that are ready for compilation without need for any manual rewrite or adaptation.

- Whether the tool is available for download and usable. In a couple of cases we could only find papers describing the tool but not a version of the implementation working on standard machines.

- An assessment of the readability of the code produced. The evaluation of this aspect-subjective to a certain extent -is based on the analysis of sample programs translated using the various tools; when the tool was not available, we analyzed translation examples discussed in the tool documentation. In each case, we tried to evaluate if the translated code is sufficiently similar to the $\mathrm{C}$ source to be readily understandable by a programmer familiar with the latter.

- Whether the tool supports unrestricted calls to external libraries, unrestricted pointer arithmetic, unrestricted gotos, and inlined assembly code.

See [1] for a discussion of other work related to C2Eif; and [3] for a comparison among Ephedra, C2J++ and C2 J.

Acknowledgements. This work was partially supported by ETH grant "Object-oriented reengineering environment".

\section{REFERENCES}

[1] M. Trudel, C. A. Furia, M. Nordio, B. Meyer, and M. Oriol, "C to O-O Translation: Beyond the Easy Stuff," in WCRE, 2012.

[2] G. C. Necula, S. McPeak, S. Rahul, and W. Weimer, "CIL: Intermediate Language and Tools for Analysis and Transformation of C Programs," in Conference on Compilier Construction, 2002, pp. 213-228.

[3] J. Martin and H. A. Müller, "Strategies for migration from C to Java," in CSMR. IEEE Computer Society, 2001, pp. 200-210.

[4] E. Tilevich, "Translating C++ to Java," in First German Java Developers' Conference Journal, Sun Microsystems Press. IEEE Computer Society, 1997.

[5] Novosoft, "C2J: a C to Java translator," http://www.novosoft-us.com/ solutions/product_c2j.shtml 2001.

[6] Tangible Software Solutions, "C++ to C\# and C++ to Java," http://www. tangiblesoftwaresolutions.com/ 


\section{APPENDIX}

\section{A. Using C2Eif with an Example}

This section demonstrates the most significant steps of using C2Eif to translate a simple $\mathrm{C}$ program into Eiffel, from the perspective of a user called Bill. Bill has come across the $\mathrm{C}$ implementation of a function index_of that takes an integer array list of length len and an integer elem, and returns the position of the first occurrence of elem in list, or -1 if elem is not found in list.

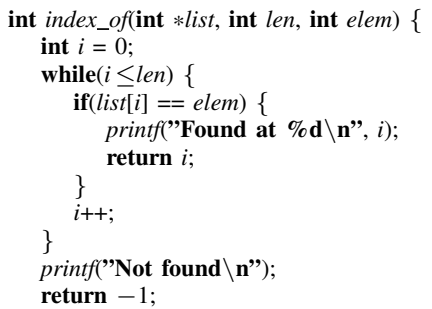

Even if the program is simple, it includes some features typical of $\mathrm{C}$ and with no direct Eiffel counterpart: arrays as pointers, control-flow breaking instructions (i.e., return inside the while loop), and calls to printf from the $\mathrm{C}$ native $\mathrm{I} / \mathrm{O}$ library. Observant readers will also notice an "off-by-one" error in the loop condition on line 3-it should be $i<$ len-that may produce a segmentation fault.

Bill, however, does not pay attention to this bug and just wants to translate index_of into Eiffel. He first transforms the C snippet using CIL; the result is a slightly less readable version of the snippet with a few (unnecessary) explicit casts (e.g., to char const $*$ for constant strings), and the access list $[i]$ to the $i$-th element of list expressed with pointer arithmetic as $*($ list $+i)$. The CIL version of the input is ready for C2Eif; Bill just specifies the input file and the output location (with more complex examples, he might have had to specify which system libraries to link) and launches the translation.

The whole translation terminates in a few instants by writing out the final output. Bill can also inspect the intermediate results after applying only some of the incremental transformations (see Section III). For example, this is the state of the program after applying the first 29 transformations:

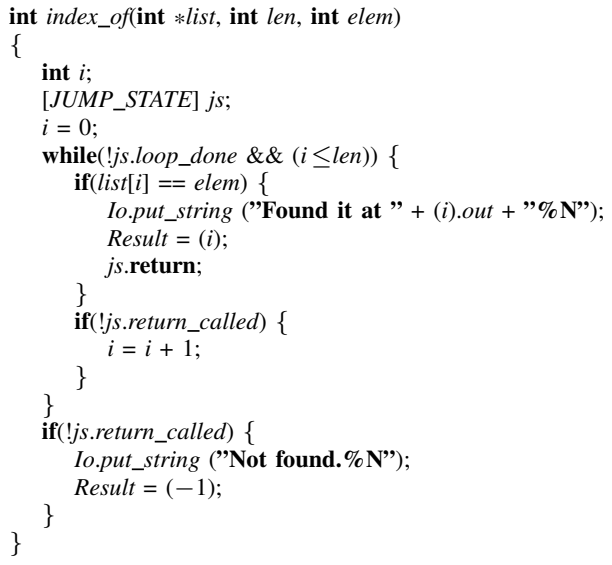

At this point in the translation, C2Eif has recognized the usages of printf and replaced them with calls to Eiffel's native put_string (lines 8 and 17); this is a special optimization for the most used $\mathrm{C}$ library functions that have counterparts in Eiffel, but C2Eif can still generate custom wrappers in Eiffel to handle calls to any native $\mathrm{C}$ library. It has used assignments to the implicit return variable Result to implement returned values in Eiffel (lines 9 and 18). It has also removed some unnecessary casts introduced by CIL and modified the condition of the while loop on line 6 to render the semantics of the control-flow breaking return inside the loop body. To this end, the translation has introduced a local variable $j s$ (line 4) of a helper class JUMP_STATE that handles usages of return, break, and continue. $\mathrm{C} 2$ Eif uses a more complex translation for generic unrestricted usages of gotos and even longjmp. Finally, C2Eif has restored the more readable syntax list $[i]$ for accessing list as an array of integers on line 7, which will be directly transliterated to Eiffel's array selector operator with the same syntax. The remaining steps of the translation mostly have to deal with syntactic sugar; for example, the while loop is converted into an Eiffel until loop, the assignment operator becomes := instead of =, and some of Eiffel's style rules are enforced.

Bill opens the final output of C2Eif with the EiffelStudio IDE and integrates it within an Eiffel application that calls index_of on a sample array my_list: CE_ARRAY [INTEGER] of size 3 (initialized with all 1's) and searches for an element with value 5: $p:=$ index_of (my_list $, 3,5)$. The usage of the translation of index_of is very natural for Bill who can use standard Eiffel syntax with minimal differences. When he launches the compiled program, Bill immediately finds the off-by-one error in the loop, because it triggers the violation of a precondition checked (introduced in the translation and checked at runtime) when accessing the array. As you can see in the screenshot of Figure 2, the source of the error is apparent in EiffelStudio's debugger, which shows that the value of $a_{-}$index is 3 but it should be strictly less than the count (i.e., the array size). With this detailed information, Bill easily fixes the translated program that can be used safely from now on.

\section{B. Demo Outline}

C2Eif's home-page at:

\begin{tabular}{|l|}
\hline http://se.inf.ethz.ch/research/c2eif \\
includes the source code, different pre-compiled versions,
\end{tabular}
translations of various applications (including $\mathrm{C}$ source code, Eiffel translations and binaries), various screenshots, and a manual with step-by-step instructions on how to use C2Eif on several examples.

The tool demonstration will be along the same lines as the example in Section $\mathrm{V}$-A but will run interactively on a realworld application and will include many more details. The demo will consists of three parts:

- An overview of the tool, where we discuss its general design principles and its overall usage workflow (as in Figure 11. 


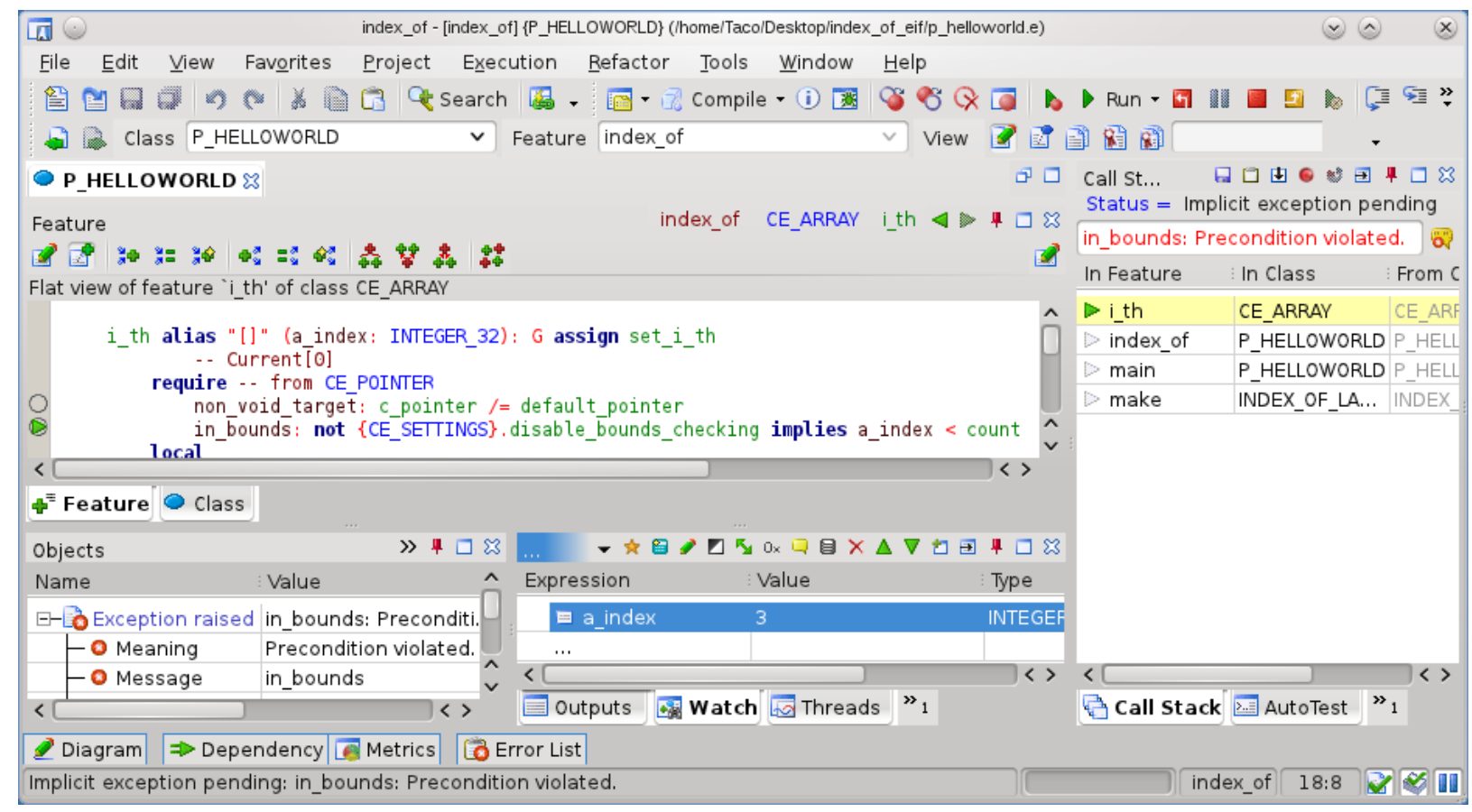

Fig. 2. Debugging a $\mathrm{C}$ function in EiffelStudio.

- A demonstration consisting of a step-by-step illustration of using C2Eif to translate xeyes from C to Eiffel ${ }^{2} \mathrm{We}$ will start the demo with $\mathrm{C}$ source code of the application freshly downloaded from open-source repositories. We will use the C2Eif GUI (shown in Figure 3) to translate the $\mathrm{C}$ code, showing the overall translation but also discussing some of its intermediate results (see Section III).

- A concluding part where we open the translated Eiffel version of xeyes with the EiffelStudio IDE, and illustrate the structure and readability of the generated code. We will also show compilation of the code, run a brief debugging session on it using Eiffel's features introduced by the translation, and modify and recompile the code on the fly.

${ }^{2}$ C2Eif's website also includes an outline of this example. 


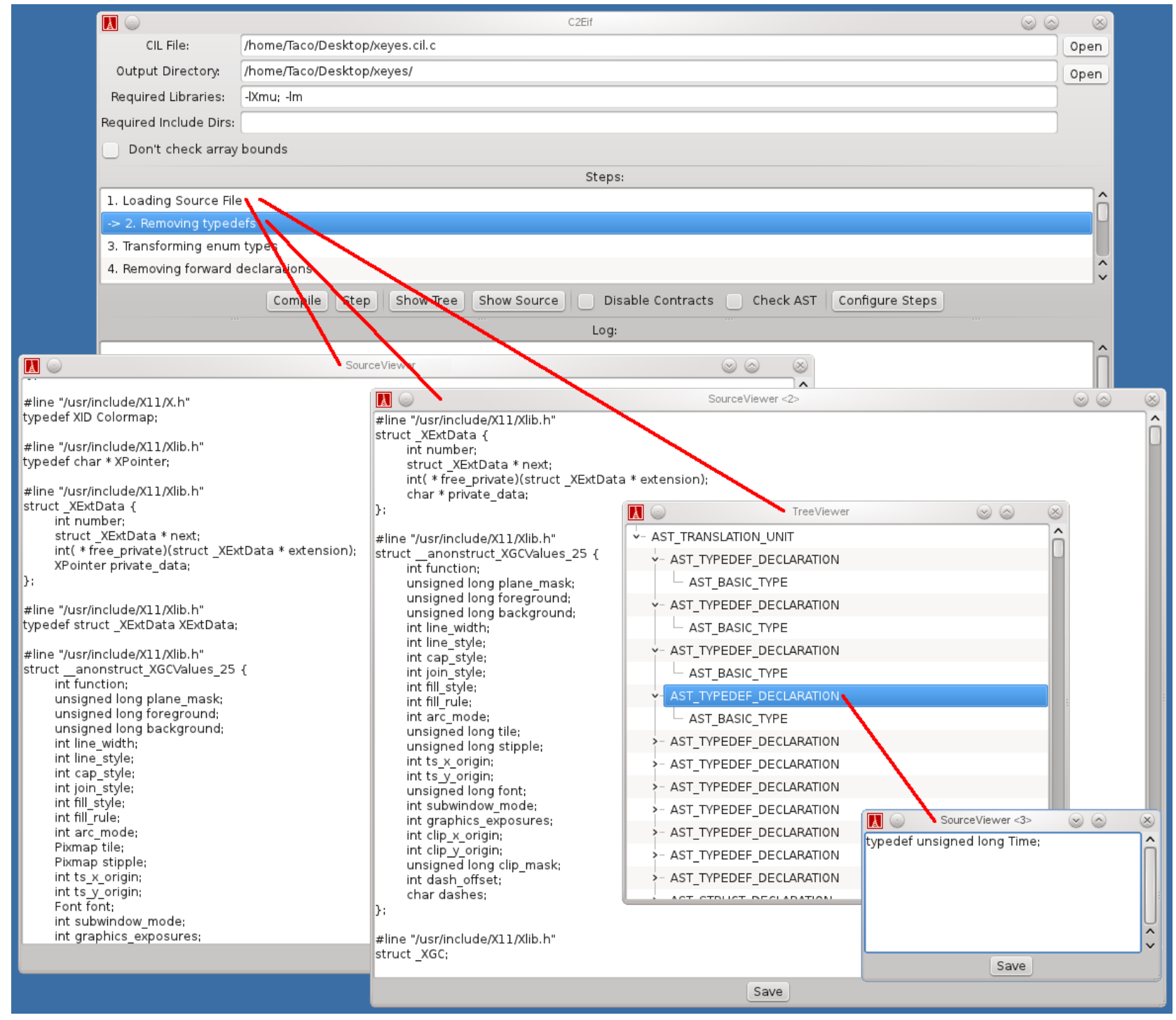

Fig. 3. Screenshot of C2Eif analyzing source code. 